\title{
Designer Antioxidants: Fortified Foods and Probiotics
}

\author{
Haseeb Ahsan ${ }^{1}$ and Rizwan Ahmad ${ }^{2 *}$ \\ ${ }^{1}$ Department of Biochemistry, Faculty of Dentistry, Jamia Millia Islamia, New Delhi, India \\ ${ }^{2}$ College of Medicine, Imam Abdulrahman bin Faisal University, Dammam, Saudi Arabia \\ *Corresponding Author: Rizwan Ahmad, College of Medicine, Imam Abdulrahman bin Faisal University, Dammam, Saudi Arabia.
}

Received: October 29, 2019; Published: November 07, 2019

DOI: 10.31080/ASNH.2019.03.0537

\begin{abstract}
Antioxidants both small molecules such GSH, thioredoxin, ascorbic acid, etc. as well as enzymatic shield of SOD, GPx, CAT, etc. counter the attack of oxidants and protect cells. Antioxidants such as tocopherols, ascorbic acid, carotenoids, flavonoids, amino acids, phospholipids, and sterols are natural antioxidants in foods. Antioxidants inhibit the oxidation of foods by scavenging free radicals, chelating prooxidative metals, quenching singlet oxygen and photosensitizers, and inactivating lipoxygenase. The importance of oxidants and antioxidants can be understood from the observation that oxidative stress is now associated with a variety of diseases including cancer, neurodegenerative diseases, diabetes and many more. Several approaches to improve human health and achieve longevity use dietary antioxidants as healthy additions in the diet. Newer approaches of designer foods such as eggs, milk and grains that are fortified with antioxidants and nutrients are currently available in the market that may be important in human health including the use of different types of probiotics, which are live microorganisms administrated in adequate amounts to confer a health benefit on the host.
\end{abstract}

Keywords: Antioxidants; Probiotics; Fortified Foods; Designer Foods; Diseases; Human Health; Lactobacillus

\section{Introduction}

Antioxidants are molecules that inhibit, decrease, delay or completely scavenge the action of free radicals and oxidants and protect the body from oxidative damage [1]. Halliwell and Gutteridge [2] defined antioxidants as "any substance that delays, prevents or removes oxidative damage to a target molecule" to include the molecules that repair the oxidative damage to the system [2,3]. Thus, effective antioxidants have the ability to delay oxidation reactions, prevent the formation of free radicals or break the autoxidation chain reaction that generates free radicals/oxidants. Oxidative/nitrosative stress results from an imbalance in oxidantantioxidant balance in favour of reactive species with the increase of (ROS) and/or reactive nitrogen species production, respectively [4]. The body's defence mechanisms against oxidative damage are operative as two main systems. The former includes removal of free radicals and reactive species by enzymes such as superoxidedismutase, catalase, glutathione peroxidase, etc. and the latter scavenges free radicals by electron donors, such as GSH, tocopherols, ascorbic acid, thioredoxin, etc. [5]. Other mechanisms include binding pro-oxidant metal ions, such as iron and copper by specific metal binding proteins such as transferrin, metallothionein, haptoglobins, caeroplasmin. The antioxidants play a major role in scavenging free radical and non-radical oxidants and protecting the cells from oxidative stress. The impairments of cellular functions caused by oxidative stress have been implicated in diseases such as neurodegenerative diseases, various cancers and aging process. Antioxidants both small molecules such GSH, thioredoxin, ascorbic acid as well as enzymes eg., SOD, GPx, CAT counter this attack and protect cellular lipids, proteins and DNA. Antioxidants such as tocopherols, ascorbic acid, carotenoids, flavonoids, amino acids, phospholipids, and sterols are natural antioxidants in foods. They inhibit the oxidation of foods by scavenging free radicals, chelating prooxidative metals, quenching singlet oxygen and photosensitizers, and inactivating lipoxygenase. Therefore, designer foods that are fortified with antioxidants and nutrients are increasing available in the market that may be important in human health including the use of different types of probiotics, which are live microorganisms administrated in adequate amounts to confer health benefit [6].

Many dietary supplements have been promoted as antioxidants, but only a few antioxidant supplements have been shown to promote health. The health effect of an antioxidant depends on the systemic bioavailability, the concentration of the compound that can be delivered to specific organ sites, and whether the antioxidant can perform the expected function. With the exception of antioxidant nutrients, many dietary antioxidants are generally less effective in combating ROS compared with the antioxidant and cytoprotective enzyme systems. Many studies have shown that taking antioxidants, especially at high doses, can lead to toxicity because of pro-oxidative activity. It would also be interesting to consider the issue of homeostasis in the redox states of tissues. ROS are also known to play physiological functions, such as being involved in killing infectious bacteria in monocytes. There are also suggestions 
that ROS plays a role in the signal transduction of many physiological functions. The physiological health requires a proper balance between ROS generation and the antioxidant systems and when ROS overcomes the antioxidant-defence systems in the body, resulting in oxidative stress [7]. The effects of food on physiological antioxidant capacities and its measurement have limitations in predicting the health effects directly in animal models and/or humans.

\section{Fortified food antioxidants}

The designer foods, also known as 'functional food' or 'fortified food', refers to the food fortified or enriched with nutrient content present in them or other complementary nutrient. These are designed to have some health benefits other than its traditional nutritional value. Designer foods are normal foods that are enriched with one or more health promoting or disease preventing substances. Most of the time the processed food is fortified with health benefiting nutrients which are already present in them in small amounts or added with complementary nutrients. A variety of foods are now available in the market that are fortified with various health promoting nutrients and antioxidants that help in the maintenance of various aspects of health ranging from the immune, visual, inflammation systems, etc. [6]. Functional foods include a wide variety of foods and food components believed to improve the overall health and wellbeing which can be produced by fortification or nutrification of conventional food. Genetically engineered foods containing higher than normal amounts of health promoting nutrients and fermented foods with live cultures are considered functional foods. Infant formula may be the first designer food as it contains nutrients for the development of brain and immune systems. The addition of docosahexaenoic acid (DHA) to health drinks for improving brain and visual development, the alteration or reduction of allergenic components in food, the use of probiotics and nucleotides to enhance immune response and sports nutrition are important examples of designer foods. Traditional and complementary medicine in various countries like China, Japan and India has the tradition of using fermented food for its health benefits, which includes red wine, yogurt, tofu, cheese, etc. [6].

Antioxidant supplements or foods containing antioxidants would greatly help in reducing oxidative damage, free radical scavenging rate, and modulating the activity of SOD and GPX in the human body. It has been reported that some probiotics result in increased activity of antioxidative enzymes or modulation of circulatory oxidative stress, which protects cells against carcinogen-induced damage. These enzymes include glutathione S-transferase, glutathione, glutathione reductase, glutathione peroxidase, superoxide dismutase and catalase. Some authors hypothesize that probiotics exert their defensive effects against oxidative stress by re-establishment of the gut flora. Some Lactobacillus spp possess antioxidant activity and are able to decrease the risk of accumulation of ROS during ingestion of food. Production of bioactive peptides has been considered an effective mode of antioxidative activity in foods containing probiotic bacteria. Peptides obtained from hydrolyzed food proteins have been shown to possess antioxidative activities, which can impart protection against the peroxidation of lipids or fatty acids. It has been observed that the gastric digestion of casein liberates small peptides with radical scavenging activity. The antioxidant ability was considered due to the presence of histidine and some hydrophobic amino acids in high concentrations. Increased antioxidant activity of milk during fermentation with commonly used dairy starter cultures, including Leuconostoc mesenteroides ssp. L. jensenii, and L. acidophilus has been observed. The intestinal Lactobacillus species are particularly important for the development of food industry due to the increasing interest of consumers for a healthy diet. During the past decade, oxidative stress and antioxidative potency have been revealed as the key features in molecular regulation of cellular stress responses. In Lactobacillus, it has been found that their antimicrobial effect is also expressed via ROS such as $\left(\mathrm{H}_{2} \mathrm{O}_{2}\right)$, superoxide anions $\left(\mathrm{O}_{2^{-}}{ }^{-}\right)$, and hydroxyl radicals $(\bullet \mathrm{OH})$, which may have an influence on the intestinal microbiota. Some lactobacilli possess antioxidative activity and are able to decrease the risk of accumulation of ROS during the ingestion of food. L. rhamnosus strain was reported to have potent antioxidative activity by downmodulating the ROS production and phagocytic capacity of neutrophils [8].

Humans have always ingested bacteria unintentionally together with food which could be harmful, but it can also be harmless "dietary bacteria" when fermented foods were consumed. In particular, lactic acid fermented foods such as yoghurt, cheese, olives may contain high amount of live bacteria and often bacteria of the Lactobacillus species are now used for probiotics. Microorganisms colonized throughout the gastrointestinal tract of human have coevolved through symbiotic relationship and influence physiology, metabolism, nutrition and immune functions of an individual. The gut microbes are directly involved in conferring protection against pathogen colonization by inducing direct killing, competing with nutrients and enhancing the response of gut-associated immune system. The damage in the microbiome (dysbiosis) is linked with several pathophysiological conditions viz. inflammatory bowel disease, cancer, obesity, allergy, and auto-immune disorders [9-15].

Probiotics are organisms or substances that contribute to intestinal microbial balance, in contrast to antibiotics that counteract microbial activity. However, the widely accepted definition is that "probiotics are live microorganisms when administrated in adequate amounts confer a health benefit on the host". Probiotics have established their efficacy as dietary factors that can regulate gastrointestinal functions, thereby imparting health benefits to consumers such as alleviation of lactose intolerance, prevention 
of diarrhoea and urogenital infections, reduction in cholesterol, modulation of the immune system, etc. Lactobacilli are the major source of probiotics and are Gram-positive bacteria which prefer anaerobic conditions, aerotolerant, fastidious, and are strictly fermentative producing lactic acid as the main product. Various species and strains of lactobacilli, namely, Lactobacillus acidophilus, Lactobacillus casei, Lactobacillus rhamnosus, and Lactobacillus helveticus are considered as successful probiotics, and are available in the market for human consumption for various health beneficial effects but their role as antioxidants is not fully understood [8].

\section{Conclusion}

Antioxidant in food sciences describe compounds that block oxidative reactions, thereby maintaining freshness and prolonging the shelf lives of food products. Dietary antioxidants and antioxidant supplements quench ROS and may prevent different chronic diseases. In addition to the essential antioxidant nutrients such as vitamins $\mathrm{E}$ and $\mathrm{C}$, there are several well-designed antioxidants and cytoprotective enzyme systems in the human body, which are more important than dietary non-nutrient antioxidants. At high concentration, many antioxidants could act as pro-oxidants, increasing oxidative stress and inducing toxicity. Humans have always ingested bacteria unintentionally together with food which could be adverse, but they could also be harmless "dietary bacteria" when fermented foods consumed. In particular, lactic acid fermented foods such as yogurt, cheese, olives contain live bacteria and often bacteria of the Lactobacillus species that are now used for probiotics. Microorganisms colonized throughout the gastrointestinal tract of humans coevolved through symbiotic relationships and influence physiology, metabolism, nutrition and immune functions of an individual.

\section{Acknowledgments}

RA is grateful to the Professor Ali Ibrahim Al-Sultan, Dean College of Medicine and Dr. Mohammad Madadin, Vice Dean of Academic Affairs for the help and support. Dr. Ahmed Al-Sunni, Chairman, Department of Physiology needs special thanks for providing necessary facilities.

\section{Conflict of Interest}

The authors declare that there is no conflict of interest.

\section{Bibliography}

1. Lobo V., et al. "Free radicals, antioxidants and functional foods: Impact on human health". Pharmacognosy Review 4.8 (2010): 118-126.

2. Halliwell B and Gutteridge JM. "The definition and measurement of antioxidants in biological systems". Free Radical Biology and Medicine 18 (1995): 125-126.

3. Halliwell B. "Antioxidant characterization. Methodology and mechanism". Biochemistry Pharmacology 49 (1995): 13411348.
4. Kurutas EB. "The importance of antioxidants which play the role in cellular response against oxidative/nitrosative stress: current state". Nutrition Journal 15.1 (2016): 71.

5. Devasagayam TPA., et al. "Free Radicals and Antioxidants in Human Health: Current Status and Future Prospects". JAPI 52 (2004).

6. Rajasekaran A and Kalaivani MJ. "Designer foods and their benefits: A review”. Food Science Technology 50 (2013): 1-16.

7. Yang CS., et al. "Antioxidants: Differing Meanings in Food Science and Health Science". Journal of Agricultural and Food Chemistry 66 (2018): 3063-3068.

8. Mishra V., et al. "Probiotics as potential antioxidants: a systematic review". Journal of Agricultural and Food Chemistry 63.4 (2015): 3615-3626.

9. Mukherjee S., et al. "Gut microbes as future therapeutics in treating inflammatory and infectious diseases: Lessons from recent findings". Journal of Nutritional Biochemistry 61 (2018): 111-128.

10. Mamantopoulos M., et al. "Inflammasomes make the case for littermate-controlled experimental design in studying hostmicrobiota interactions". Gut Microbes 9 (2018): 374-381.

11. Richard ML and Sokol H. "The gut mycobiota: insights into analysis, environmental interactions and role in gastrointestinal diseases". Nature Reviews Gastroenterology and Hepatology 16 (2019): 331-345.

12. Halliwell B., et al. "The characterization of antioxidants". Food and Chemical Toxicology 33.7 (1995): 601-617.

13. Halliwell B. "Biochemistry of oxidative stress". Biochemical Society Transactions 35 (2007): 1147-1150.

14. Halliwell B and Gutteridge JM. "Free radicals in biology and medicine". Oxford University Press USA, (2015).

15. Halliwell B. "How to characterize an antioxidant: an update". Biochemical Society Symposia 61 (1995): 73-101.

\section{Volume 3 Issue 12 December 2019} (C) All rights are reserved by Haseeb Ahsan and Rizwan Ahmad. 